\title{
IN VIVO ANIMAL MODELS IN PERIODONTAL RESEARCH - FOCUS ON RODENTS
}

\author{
E. I. FIRKOVA ${ }^{1} \&$ TS. CHAPRAZOV ${ }^{2}$ \\ ${ }^{1}$ Department of Periodontology and Oral Diseases, Faculty of Dental Medi- \\ cine, Medical University, Plovdiv, Bulgaria; ${ }^{2}$ Department of Veterinary Sur- \\ gery, Faculty of Veterinary Medicine, Trakia University, \\ Stara Zagora, Bulgaria
}

\section{Summary}

Firkova, E. I. \& Ts. Chaprazov, 2021. In vivo animal models in periodontal research - focus on rodents. Bulg. J. Vet. Med., 24, No 2, 167-175.

Periodontal research has developed very fast in the last two decades. Although at this stage of science a lot of genetic and molecular-based trials are performed in order to elucidate the complex etiology, pathophysiology, biofilm-host interactions and responses on genetic and cellular level, in vivo animal models are still used. In many ways, in vivo experiments are superior to in vitro tests when the dynamics of the immune-inflammatory nature of the periodontal disease and peri-implantitis and the specific healing of soft and hard tissues is concerned. Screening the efficacy, mechanisms of action and application of different biomaterials requires in vivo experiments, before the data translation to clinical settings. A number of small animals like rodents and large species like dogs and nonhuman primates are involved in periodontal research. As live creatures are used, the design of the studies must be well defined, with regard to the type of the animals, most suitable for the tested hypothesis, observation period, sample size, study power, critical size defects, and specific testing sites.

Key words: animal models, biomaterials, periodontal research, regeneration

\section{INTRODUCTION}

Regenerative periodontology and implant dentistry has developed very fast in the last two decades, with a variety of new biomaterials and approaches being introduced, which claim sufficient regenerative and healing potential. In vivo animal models are necessary and still stay relevant in modern dental research. Although a lot of research is accomplished using in vitro systems, animal experiments are still the most important step and the essential link between the hypothesis and the human patients. Usually after a positive in vitro testing for a certain hypothesis, evaluation of proof-of-principle concepts, safety and unwanted reactions are performed in preclinical studies before proceeding to clinical testing.

Staying up-to-day with modern principles and requirements for proper design of 
the scientific projects, this review aims at general to summarise and outline basic in vivo models, used for preclinical studies in periodontology and implant dentistry. It is also focused on experimental procedures and the surgical techniques used to test bone grafting biomaterials in rat model.

\section{GENERAL OVERVIEW}

Animal experiments date back to the earliest days of research. A recent PubMed survey yielded more than 2000 peerreviewed articles in which various animal models had been used for periodontal or peri-implant regeneration and wound healing studies (Kantarci et al., 2015). The first animal models were used to find out the relationship between periodontal infection and the host response (Hamp et al., 1972; Hamp \& Lindberg, 1977; Hugoson \& Schmidt, 1978). Many studies were conducted to define the complex etiology and pathogenesis of periodontal diseases. Although there are successful and relevant in vitro models, they cannot explain in details, for example, the specific host-parasite interactions. In vitro methods can never fully reproduce the complexity of in situ biology. There are some topics that can be elucidated only in animal models, e.g. wound healing and regeneration process in the affected periodontal tissues. The regeneration after periodontal surgery is a slow, continuous process, involving epithelial cells, connective tissue, periodontal ligament and the alveolar bone. The effects of different biomaterials, used to substitute the bone loss or to enhance the healing process in soft tissues, or possible side effects, can be validated only through well designed and performed animal studies (Sculean et al., 2015). Actually the European Medi- cines Agency and the US Food and Drug Administration often require a series of targeted pre-clinical evaluations before human trials are started.

Knowing the complexity of periodontal biology, microbial biofilm-host interactions and principles of soft tissue healing and alveolar bone regeneration, animal models are necessary and serve as standard for successful translation of regenerative biomaterials to the clinical setting.

\section{ANIMAL SPECIES USED IN PERIO- DONTAL AND BIOMECHANICAL RESEARCH}

Evaluation of bioactivity, biocompatibility, toxicity, potential adverse reactions or side effects and efficacy of a biomaterial intended to enhance bone formation requires models, closer to humans. A preclinical in vivo model should be based on similarities between the processes studied in animals and humans in health and disease, so that the data could be extrapolated to the human population. The ideal in vivo model should be performed with the phylogenetic species with anatomic similarities, biochemical and physiological mechanisms closest to those in humans (Oz \& Puleo, 2011).

Multiple animal species, including mice, rats, rabbits, dogs, pigs and nonhuman primates have been used for those purposes. The selection of animal species must be based mainly on the research question or on the disease model.

The dental anatomy of larger species dogs, sheep, miniature pigs, nonhuman primates - resembles more closely the human dentoalveolar structures. Beagle dogs are among the most used models to study naturally occurring gingivitis and periodontitis and later - peri-mucositis 
and peri-implantitis (Klinge et al., 2005; Lin et al., 2017). Histological and radiographic observations revealed in details the destructive changes in epithelial and connective tissue attachment and cementum and the patterns of alveolar bone resorption after ligature-induced periodontitis (Nyman et al., 1986) and periimplantitis. The studies with dogs have been considered also as the major in vivo testing system for new regenerative techniques or devices. Some of the earliest evidence for the efficacy of guided tissue regeneration came from dogs (Nyman et al., 1980). During the 1980s and 1990s, dog studies were extremely popular for testing of graft materials, resorbable and nonresorbable membranes, root surface conditioners (Levy et al., 1981; Fleischer et al., 1988; Magnusson et al., 1990; Pontoriero et al., 1992). Later, growth factors like platelet-derived growth factor, insulin like growth factor, as well as various extracellular matrix proteins for stimulation of new attachment formation was tested first on dogs (Lynch et al., 1991; Cho et al., 1995; Iqbal \& Bamaas, 2001). However, in recent years, the use of dog models has been substantially decreased for several reasons - ethical considerations; replacement with miniature pigs or small animal models.

Miniature pigs have been extensively used in biomedical research dealing with dermal wound healing and artificial organs. Some of pigs develop human diseases, such as diabetes, with the same pathophysiological processes involved and therefore, they are a very good model for studying systemic diseases in the field of periodontal medicine (Kalkwarf \& Krejci, 1983; Van Dorp et al., 2002). The advantages of using a miniature pig model for dental and orofacial research were presented in an excellent review by Wang et al. (2007). Although the periodontium of those pigs shows many similarities to that of humans and therefore they are suitable models for different investigations, to our knowledge pigs are still not commonly used in Bulgaria.

The major limitations of using large animals are that they are expensive; require specialised breeding and maintenance facilities and highly qualified staff and laboratories. There is also an increased risk of post-operative complications and the possibility of losing test animals in the course of the experiment.

The use of small animals (rodents, rabbits) has several advantages. The animals are of small size, which means less space for housing, easier breeding, facilities, etc. They are cheap which allows to include large number of animals for collecting sufficient data for analyses. Small animals are cost-effective also because they are easier to handle - the surgery procedures are easier and post-surgery recovery is faster; less complications can be expected compared to large animals. Small animals are more resistant to infections which reduces the risk of losing experimental animals during the course of the experiment.

Rodents are widely used animals in periodontal research. Rodents are suitable models for investigation of the dynamics of soft- and hard tissue interactions, different pathways of regulation of inflammation, signal molecules, bone turnover and regeneration, etc. They provide several advantages for evaluation of microbial and host responses. Rodents have 1 incisor and 3 molars in each quadrant. After placement of a silk ligature around a tooth and plaque accumulation, the development of periodontal disease can be evaluated. Actually rodents are used mainly to study the host-parasite interactions 
(Graves et al., 2008, 2012; de Molon et al., 2014). Rats are often infected with human periodontopathogens in order to document their virulence factors (Klausen, 1991). Induced periodontal disease is subjected to detailed microbiological and immunological studies. Antibodies against mice antigens are available almost as human panels, which allows performing different immunohistochemical investigations. The mouse genome has been sequenced, therefore the specific role of different genes in the regulation of inflammatory and healing process can be assessed. The mouse calvaria model is well-known. The experiments can provide data on the efficacy of many molecules and biomaterials, used for periodontal regeneration (Lindhe et al., 1993; Stavropoulos et al., 2003, Silva et al., 2017). Mice are convenient models to study the evolution of inflammatory and immune events. After injection of microorganisms, fast expression of proinflammatory cytokines and recruitment of neutrophil granulocytes is observed within 24 hours (Graves et al., 2008). Bone resorption can be detected as soon as 3 to 5 days, depending on the dose of the initiating stimulus (Li et al., 2002).

Rabbits are also good small-sized animals to study the impact of inflammation on periodontal and peri-implant wound healing and regeneration. A ligature, fixed around teeth or implants, does not induce by itself a periodontal inflammation. This fact allows the researchers to define clearly the specific effects of periodontal pathogens. In contrast, a ligature placed around mice and rats' teeth induces periodontal inflammation by conversion of their own commensal flora into a pathogenic one. Hasturk et al. (2006) were able to induce a predictable and reproducible periodontitis in rabbits by us- ing silk ligatures together with topical application of Porphyromonas gingivalis. They observed clinical, radiographic and histologic signs of periodontitis, very similar to those in humans. Stopping the application of the pathogen at the end of the 6-week experiment did not result in spontaneous resolution of inflammation or healing. Using this model, a lot of compounds and their efficacy were tested with clinical implications of the results in humans (Hasturk et al., 2007; 2009).

Rabbits are used also for studying osseointegration of implants. Most of these techniques involve the long bones as orthotopic testing sites (Indjova et al., 2014). These studies test not only the integration of new materials and surface designs, but also the effect of confounding factors on bone healing and implant integration such as bisphosphonates (Chacon et al., 2006).

\section{EXPERIMENTAL MODELLING: RATIONALE, RELEVANCE, SIZE ISSUES}

Planning an in vivo experiment for the needs of periodontal research, requries very careful consideration of all details, having in mind that live creatures are involved. The goals should be clearly established. Correct calculation of sample size and study power are very important, so that there is enough data for statistical analyses without unnecessary use of animals. For testing the efficacy, mechanisms of action, bone regeneration and soft tissue response and healing of different biomaterials is possible using 2 possible testing sites - heterotopic or orthotopic. The choice of test site is made on the specific hypothesis tested.

Heterotopic sites are within the soft tissues of the animal - intramuscular or 
epimuscular, subcutaneous or intrafatty. Heterotopic sites are used when pharmacological and pharmacokinetic properties, as well as possible side effects and toxicity of different medications are evaluated (Schuh, 2008).

Orthotopic sites are used mainly when regenerative capacity of biomaterials is tested. Orthotopic sites can be intraosseous or periosseous or extra-skeletal. Intraosseous/periosseous site can be created in different locations - calvarium, mandible, long bones, sinus (Klinge \& Johnson, 2012).

Selection of this model should be based on many factors (Stavropoulos et al., 2015):

- Mechanism of action (whether the biomaterial has osteoconductive or osteoinductive properties);

- The physical properties of the material (small particles, block, gel, membrane);

- The intended clinical application (fill in or cover defects).

Probably the most useful pre-clinical model for bone regeneration materials is the intraosseous site model with a creation of so-called "critical size" defects. The latter are osseous defects that do not heal completely during the lifetime of the animal unless subjected to an intervention (Smitz \& Hollinger, 1986). This model involves creation of cylindrical defects on the calvaria of small animals - rats, mice and rabbits. Different sizes of defects are used, depending on the species and the age. The critical size in mice is $3-5 \mathrm{~mm}$, in rats: $5-8 \mathrm{~mm}$ and $15 \mathrm{~mm}$ in rabbits. Actually even a very small defect size will be critical, provided that the experiment is of short duration but reasonable interpretation of results can be made only if the defects have relevant dimensions, so that the biomaterial can enhance itself the bone formation.

The procedure of creating the critical size defect in calvaria of rodents is standardised (Schmitz \& Hollinger, 1986; Cooper et al., 2010). Usually 2 symmetrical circular bicortical defects are created in the parietal and/or the occipital bone, so that the test and control sites can be observed being under the same conditions. Access to the calvarium is made by midline or lateral skin incisions, extending from the nasofrontal area to the occipital protuberance. After skin elevation and incision of the subcutaneous fascia, the bone is exposed by a blunt dissection. The anatomical characteristics of the calvarium allows easy creation of standardised, reproducible defects, usually made by trephine bur. The procedure must be done carefully, with respect to the animal following all surgical principles for good practice. Drilling of bone is performed by a trephine and traditionally $5 \mathrm{~mm}$ round defects are created. Care must be taken not to damage the underlying dura mater, blood vessels or cranial sinus. The bone disk must be removed very carefully, in order not to tear the subjacent cranial structures. If a large damage occurs in mice calvaria, it will result in protrusion of brain into the created bone defect, thus eliminating the test/experimental space. Moreover, the lack of dura continuity decreases the bone formation; when it is intact the defect show complete fill in immature rats (Mossaz \& Kokich, 1981). After the procedure is performed, the periosteum, fascia and skin are repositioned and tightly sutured. It is important to note that dura has the ability to stimulate osteogenesis by expression of fibroblast growth factor- 2 and transforming growth factor- $\beta$. In case of testing new biomaterials is reasonable to use barrier mem- 
brane to limit the ingrowth of soft connective tissue and maintain a high concentation of locally produced osteogenic factors (Dahlin et al., 1991).

The advantages of this "critical size" calvarial defects include the easy access and handling procedures; possibility for radiographic and histological analyses. A possible limitation is that there is no option for evaluating the biological response of the implanted biomaterial to physiological biomechanical loading.

The model is well defined, in means that it is known how long time it takes for the cortical defect to recover. It has been proved that a period of 4 to 8 weeks is necessary for the bone to regenerate to reach a plateau; beyond that period no significant bone formation can be expected (Gosain et al., 2000).

Possible outcome variables in this model include (Stavropolous et al., 2015):

- Extent of defect closure (length and area);

- Thickness of the osseous bridge;

- Relative and absolute volumes of tissue components (bone, connective tissue and biomaterial).

However, this approach probably will change in the near future. Some authors (Cooper et al., 2010) suggest that the term "critical size defect" should be discontinued, because the outcomes (like quality of bone formation) can be more accurately evaluated by microcomputer tomography or other advanced techniques in live animals. Until such new approaches are widely accepted and approved as relevant for clinical interpretations, the critical size model is still the gold standard for bone healing strategies.

\section{CONCLUSIONS}

In vivo small animal models are essential for periodontal research. Testing the efficacy and specific mechanisms of action of different biomaterials used for periodontal regeneration and wound healing is necessary to translate the knowledge to humans. No animal model can completely recreate the specific characteristics of the human periodontal complex. That is why the design of such models must be carefully cleared in details, in terms of most suitable for the main goal (research question or disease model) species, observation period, sample size, study power, critical size defects, and specific testing sites. Post-operative care for the animals must be performed following the best practice.

\section{REFERENCES}

Chacon, G. E., E. A. Stine, P. E. Larsen, F. M. Beck \& E. A. McGlumpy, 2006. Effect of alendronate on endosseous implant integration: An in vivo study in rabbits. Journal of Oral and Maxillofacial Surgery, 64, 1005-1009.

Cho, M. I., W. L. Lin \& R. J. Genco, 1995. Platelet-derived growth factor-modulated guided tissue regenerative therapy. Journal of Periodontology, 66, 522-530.

Cooper, G. M, M. P. Mooney, A. K. Gosain, P. G. Campbell, J. E. Loose \& J. Huard, 2010. Testing the "critical-size" in calvarial bone defects: Revisiting the concept of a critical-size defect (CSD). Plastic Reconstructive Surgery, 125, 1685-1692.

Dahlin, C., P. Alberius \& A. Lindhe, 1991. Osteopromotion for cranioplasty: An experimental study in rats. Journal of Oral and Maxillofacial Surgery, 51, 892-987.

De Molon, R. S., E. D. de Avida, A. Nogueira, J. A. C. de Souza, M. Campos, C. R. de Andrade \& J. A. Cirelli, 2014. Evaluation of the host response in various models of 
induced periodontal diseases. Journal of Periodontology, 85, 465-477.

Fleischer, N., H. de Wall \& A. Bloom, 1988. Regeneration of lost attachment apparatus in the dog using Vicryl absorbable mesh (Polyglactin 910). International Journal of Periodontics and Restorative Dentistr, 8, 44-55.

Gosain, A. K., L. S. Song, P. R. Yu \& B. J. Mehrara, 2000. Osteogenesis in cranial defects: reassessment of the concept of the critical size and the expression of the TGFbeta isoforms. Plastic and Reconstructive Surgery, 106, 360-371.

Graves, D. T., D. Fine, Y. T. Teng, T. van Dyke \& G. Hajishengalis, 2008. The use of rodent models to investigate host-bacteria interactions related to periodontal diseases. Journal of Clinical Periodontology, 35, 89-105.

Graves, D. T., J. Kang, O. Andriankaja, K. Wada \& C. Rossa Jr, 2012. Animal models to study host-bacteria interactions involved in periodontitis. Frontiers of Oral Biology, 15, 117-132.

Hamp, S. E., J. Lindhe \& H. Loe, 1972. Experimental periodontitis in beagle dogs. Journal of Periodontal Research, 7, $13-$ 14.

Hamp, S. E. \& R. Lindberg, 1977. Histopathology of spontaneous periodontitis in dogs. Journal of Periodontal Research, 12, 46-54.

Hasturk, H., A. Kantarci, N. Ebrahimi, C. Andry, M. Holick, V. L. Jones \& T. E. Van Dyke, 2006. Topical He antagonist prevents periodontitis in rabbit model. Infection and Immunity, 74, 2402-2414.

Hasturk, H., A. Kantarci, E. Goguet-Surmenian, A. Blackwood, C. Andry, C. N. Serhan \& T. E. Van Dyke, 2007. Resolving E1 regulates inflammation at the cellular and tissue level and restores tissue homeostasis in vivo. Journal of Immunology, 179, 7021-7029.

Hasturk, H., E. Goguet-Surmenian, A. Blackwood \& C. Andry, 2009. 1-tetradecanol complex: Therapeutic actions in experimental periodontitis. Journal of Periodontology, 80, 1103-1113.

Hugoson, A. \& G. Schmidt, 1978. Influence of plaque control on the healing of experimentally-induced bone defects in the dog. Journal of Periodontology, 49, 135-141.

Indjova, J., D. Sivrev, Kh. Fakih, M. Paskalev \& Ts. Chaprazov, 2014. Repair of artificial bone defects using guided bone regeneration with BioOss and Enamel Matrix Derivate. I. Light microscopic study on long bones in rabbits. Bulgarian Journal of Veterinary Medicine, 17, 134-146.

Iqbal, M. K. \& N. Bamaas, 2001. Effect of enamel matrix derivate (EMDOGAIN) upon periodontal healing after replantation of permanent incisors in beagle dogs. Dental Traumatology, 17, 36-45.

Kalkwarf, K. L. \& R. F. Krejci, 1983. Effect of inflammation on periodontal attachment levels in miniature swine with mucogingival defects. Journal of Periodontology, 54, 361-364.

Kantarci, A., H. Hasturk \& T. E. van Dyke, 2015. Animal models for periodontal regeneration and peri-implant responses. $\mathrm{Pe}$ riodontology 2000, 68, 66-82.

Klausen, B., 1991. Microbiological and immunological aspects of experimental periodontal disease in rats: A review article. Journal of Periodontology, 62, 59-73.

Klinge, B., M. Hultin \& T. Berglundh. Periimplantitis, 2005. The Dental Clinics of North America, 49, 661-676.

Klinge, B. \& J. Johnsson, 2012. Animal models in oral health science. In: Handbook of Laboratory Animal Science, $2^{\text {nd }}$ edn, eds J. Hau \& G. L. Shapiro, CRC Press, pp. 387-418.

Levy P., A. Nevins \& R. La Porta, 1981. Healing potential of surgically-induced periodontal osseous defects in animals using mineralized collagen gel xenografts. Journal of Periodontology, 52, 303-306.

Li, L., A. Khansari, L. Shapira, D. T. Graves \& S. Amar, 2002. Contribution of inter- 
leukin-11 and prostaglandin(s) in lipopolysaccharide-induced bone resorption in vivo. Infection and Immunity, 70, 39153922.

Lin, X., T. Liu, G. Wu \& Y. Zheng, 2017. Peri-implantitis induced by stainless ligature in beagle dogs. The International Journal of Periodontics and Restorative Dentistry, 37, 170-179.

Lindhe, A., C. Thoren, C. Dahlin \& E. Sandberg, 1993. Creation of new bone by an osteopromotive membrane technique: An experimental study in rats. Journal of Oral and Maxillofacial Surgery, 51, 892-897.

Lynch, S. E, G. R. de Castlla, R. C. Williams, T. H. Howell, M. S. Reddy \& H. N. Antoniades, 1991. The effect of short-term application of a combination of plateletderived and insulin-like growth factors on periodontal wound healing. Journal of $\mathrm{Pe}$ riodontology, 62, 458-467.

Magnusson, I., W. V. Stenberg, C. Batich \& J. Egelberg, 1990. Connective tissue repair in circumferential periodontal defects in dogs following use of a biodegradable membrane. Journal of Clinical Periodontology, 17, 243-248.

Mossaz, C. F. \& V. G. Kokich, 1981. Redevelopment of the calvaria after partial craniectomy in growing rabbits: the effect altering dural continuity. Acta Anatomica, 109, 321-328.

Nyman, S., G. Sahed, I. Ericsson, J. Gottlow \& T. Karring, 1986. Role of "diseased" root cementum in healing following treatment of periodontal disease. An experimental study in dog. Journal of Periodontal Research, 21, 496-503.

Nyman, S., T. Karring, J. Lindhe \& S. Planten, 1980. Healing following implantation of periodontitis-affected roots into gingival connective tissue. Journal of Clinical Periodontology, 7, 394-401.

Oz, H. S. \& D. A. Puleo, 2011. Animal models for periodontal disease. Journal of Biomedicine and Biotechnology, 11, 754-857.
Pontoriero, R., S. Nyman, I. Ericsson \& J. Lindhe, 1992. Guided tissue regeneration in surgically produced furcation defects. An experimental study in the beagle dogs. Journal of Clinical Periodontology, 19, 159-163.

Sculean, A., I. L. C. Chapple \& W. Giannobile, 2015. Wound models for periodontal and bone regeneration: The role of biological research. Periodontology 2000, 68, 7-20.

Silva, E. C., S. V. Omonte, A. G. Martins, H. H. de Castro, E. G. Zenobio, P. A. Oliveira, M. C. R. Horta \& P. E. A. Souza, 2017. Hyaluronic acid on collagen membranes: An experimental study in rats. Archives of Oral Biology, 73, 214-222.

Smitz, J. P. \& J. O. Hollinger, 1986. The critical size defect as an experimental model for craniomandibulofacial nonunions. Clinical Orthopaedics and Related Research, 205, 299-308.

Stavropoulos, A., L. Kostopollous, J. R. Nyengaard \& T. Karring, 2003. Deproteinized bovine bone (Bio-Oss) and bioactive glass (Biogran) arrest bone formation when used as an adjunct to guided tissue regeneration: An experimental study in the rat. Journal of Clinical Periodontology, 30, 636-643.

Stavropoulos, A., A. Sculean, D. D. Bosshardt, D. Buser \& B. Klinge, 2015. Pre-clinical in vivo models for the screening of bone biomaterials for oral/craniofacial indications: Focus on small-animal models. $P e$ riodontology 2000, 68, 55-65.

Van Dorp, A. G., M. C. Verhoeven, H. K. Koerten, T. H. van Der Nat-Van Der Meji, C. A. Van Blitterswijik \& M. Ponec, 2002. Dermal regeneration in full thickness wounds in Yucatan miniature pigs using a biodegradable copolymer. Wound Repair and Regeneration, 6, 556-568.

Wang, S., Y. Liu, D. Fang \& S. Shi, 2007. The miniature pig: A useful large animal model for dental and orofacial research. Oral Diseases, 13, 530-537. 


\section{Correspondence:}

\section{Elena Firkova}

Department of Periodontology and Oral Diseases, Faculty of Dental Medicine,

Paper received 19.04.2019; accepted for publication 15.10.2019

Plovdiv, Bulgaria

e-mail: elenafirkova@yahoo.com 